\title{
Efficacy of preemptive analgesia on acute postoperative pain in children undergoing major orthopedic surgery of the lower extremities
}

This article was published in the following Dove Press journal: Journal of Pain Research

\section{Do-Hyeong Kim \\ Namo Kim \\ Jae Hoon Lee \\ Minju Jo \\ Yong Seon Choi}

Department of Anesthesiology and Pain Medicine, Anesthesia and Pain Research Institute, Yonsei University College of Medicine, Seoul, Republic of Korea
Correspondence: Yong Seon Choi Department of Anesthesiology and Pain Medicine, Anesthesia and Pain Research Institute, Yonsei University College of Medicine, 50-I, Yonsei-ro, Seodaemun-gu, Seoul 03722, Republic of Korea

$\mathrm{Tel}+82222282412$

Fax +82 222277897

Email yschoi@yuhs.ac
Background: Children undergoing major orthopedic surgery of the lower extremities can experience severe postoperative pain; yet, the ideal postoperative pain management strategy is unknown. Thus, in this patient population, we investigated the effect of intraoperative epidural infusion of local anesthetic on acute postoperative pain and analgesic consumption.

Patients and methods: Patients $(\mathrm{N}=50,3-12$ years) randomly received either ropivacaine $0.15 \%$ (preemptive group) or normal saline (control group) as an initial bolus of $0.2 \mathrm{~mL} / \mathrm{kg}$, followed by continuous infusion of $0.15 \mathrm{~mL} / \mathrm{kg} / \mathrm{h}$ throughout surgery. Following surgery, patientcontrolled epidural analgesia with ropivacaine $0.1 \%$ was provided. The main study outcomes were the revised Face, Legs, Activity, Cry, and Consolability pain scores, epidural ropivacaine consumption, and additional analgesic requirements during the first 48 hours postoperatively. Results: Forty-seven patients completed the study, 23 in the preemptive group and 24 in the control group, respectively. The revised Face, Legs, Activity, Cry, and Consolability pain scores were significantly lower in the preemptive group only at 30 minutes after postanesthesia care unit arrival and 6 hours after surgery (median difference $-1.0,95 \% \mathrm{CI}-2.0$ to $-1.0, P=0.001$ and median difference $-2.0,95 \% \mathrm{CI}-3.0$ to $-1.0, P=0.005$, respectively). However, they were not significantly different between the groups at 12,24 , and 48 hours postoperatively. Epidural ropivacaine consumption and additional analgesic requirements throughout 48 hours postoperatively were not significantly different between the groups.

Conclusion: Intraoperative epidural infusion of ropivacaine did not demonstrate preemptive analgesic efficacy within 48 hours postoperatively in children undergoing extensive lower limb orthopedic surgery.

Keywords: epidural analgesia, postoperative analgesia, orthopedics, pediatrics

\section{Introduction}

Children with musculoskeletal pathology often require extensive surgical interventions to correct deformities and improve function. Such extensive surgery renders significant tissue damage and intense postoperative pain. ${ }^{1}$ Inadequate treatment of postoperative pain can lead to poor outcomes; thus, postoperative pain management is an essential part of perioperative care in pediatric patients undergoing orthopedic surgery. ${ }^{2,3}$ However, the optimal strategy for postoperative pain management in this patient population is unknown.

One potential strategy is preemptive analgesia. This intervention is designed to reduce acute postoperative pain and minimize the development of chronic pain conditions. Preemptive analgesia prevents central sensitization by interrupting the transmission of the peripheral afferent nociceptive barrage to the spinal cord. ${ }^{4}$ 
Although numerous experimental studies have demonstrated the efficacy of preemptive analgesia on postoperative pain, results of clinical studies are controversial. ${ }^{4,5}$ For example, unlike in the study using local anesthetic only for epidural infusion, no difference in postoperative pain relief between presurgical and postsurgical continuous epidural analgesia was observed in the study using local anesthetic and morphine mixture for epidural infusion. ${ }^{6,7}$ This could be because the use of morphine, which has a sedative effect, masked any preemptive analgesic effects. In addition, these conflicting results may result from variations in the relative timing of treatment initiation, ${ }^{4,8}$ or from incomplete afferent blockade using systemic analgesics or local anesthetics. ${ }^{9}$

In children undergoing extensive lower limb orthopedic surgery, postoperative epidural analgesia is beneficial. ${ }^{10}$ For these patients, considering that pathological changes that could aggravate acute pain and contribute to pain chronicity occur in the central nervous system, preemptive analgesia including intraoperative epidural infusion of local anesthetic may sufficiently protect the central nervous system from nociceptive input throughout surgery and subsequently reduce postoperative pain. ${ }^{8}$ Previously, Ong et al reported that preemptive epidural analgesia improves acute postoperative pain $;{ }^{11}$ however, the effect of preemptive epidural analgesia on acute postoperative pain in pediatric orthopedic surgery is unknown.

Thus, the primary aim of this study was to evaluate whether intraoperative epidural infusion of local anesthetic would improve acute postoperative pain in pediatric patients undergoing major orthopedic surgery of the lower extremities. We also investigated postoperative analgesic consumption, including epidurally administered local anesthetic and systemic analgesics.

\section{Patients and methods}

The study protocol was approved by the institutional review board of the Yonsei University Health System, Seoul, South Korea (\#4-2016-0322) and registered at ClinicalTrials.gov (NCT02813018). After receiving written informed consent from the parents or legal guardians, we enrolled 50 patients (range, 3-12 years) who were scheduled to undergo singleevent multilevel surgery that included at least one bony surgical procedure and/or reconstructive hip surgery for gait improvement between July 2016 and July 2017. Exclusion criteria were as follows: 1) known or suspected coagulopathy, 2) congenital anomalies or signs of infection at the site of the proposed epidural block, 3) allergy to the study drugs, and 4) severe-profound cognitive impairment. Enrolled patients were randomized to receive intraoperative epidural infusion of either ropivacaine $0.15 \%$ (preemptive group) or normal saline (control group). Randomization was performed using a computer-generated randomization sequence (http://www. randomizer.org) by an investigator not involved in patient care or perioperative assessment. Allocation results were concealed in sealed opaque envelopes that were given to an anesthesia nurse not involved with the study. The nurse prepared ropivacaine $0.15 \%$ or normal saline in identical syringes under sterile conditions according to the allocation. Consequently, the surgeons, attending anesthesiologists, investigators, nursing staff, and patients' parents were blinded to the group assignment throughout the study.

\section{Intraoperative management}

No premedication was administered. Upon arrival to the operating room, standard monitoring and measurement of the bispectral index were initiated. Anesthesia was induced with propofol $1.0-2.0 \mathrm{mg} / \mathrm{kg}$ and fentanyl $1.0 \mu \mathrm{g} / \mathrm{kg}$, followed by rocuronium $0.6 \mathrm{mg} / \mathrm{kg}$ for muscle relaxation to facilitate tracheal intubation. A radial artery catheter was then placed for continuous arterial pressure monitoring. Anesthesia was maintained with sevoflurane at a 0.7-1.5 ageadjusted minimum alveolar concentration in a 50\% oxygenair mixture, with a target bispectral index between 40 and 60. Sevoflurane was adjusted to maintain the mean arterial pressure (MAP) and heart rate (HR) within 80\%-120\% of preoperative values. Controlled ventilation was performed to maintain an end-tidal carbon dioxide tension of 35-40 mmHg. Hypotension (MAP $<80 \%$ of baseline) persisting for 5 minutes was treated with normal saline boluses and, if required, ephedrine or phenylephrine. Bradycardia (HR $<60$ beats/min) was treated with atropine. After surgery and cast application, neuromuscular blockade was antagonized with neostigmine and glycopyrrolate, and the trachea was extubated when patients were fully awake and breathing adequately. All patients were transferred to the postanesthesia care unit (PACU).

\section{Intervention}

After tracheal intubation, patients were placed in the lateral decubitus position. An 18-gauge Tuohy needle was inserted at the L3-4 or L4-5 interspace using the midline approach. A 20-gauge epidural catheter was advanced up to $3 \mathrm{~cm}$ cephalad into the epidural space, which was identified by the loss-ofresistance-to-saline technique. The catheter was aspirated to exclude intravenous or intrathecal placement, and after securing the catheter, patients were returned to the supine position 
for surgical preparation. Patients then received a bolus of 0.2 $\mathrm{mL} / \mathrm{kg}$ of the prepared solution (ropivacaine $0.15 \%$ or normal saline) through the catheter, and the same solution was infused at a rate of $0.15 \mathrm{~mL} / \mathrm{kg} / \mathrm{h}$ using a syringe pump 15 minutes before skin incision until the end of the surgery. The mean duration of infusion was 301.6 minutes. The maximum intraoperative infusion rate in patients exceeding $30 \mathrm{~kg}$ was $4.5 \mathrm{~mL} / \mathrm{h}$. The mean operation time was 286.6 minutes. At the end of surgery, epidural infusion was stopped, followed by the same epidural patient-controlled analgesia (PCA). The PCA device containing ropivacaine $0.1 \%$ in normal saline solution with a total volume of $9 \mathrm{~mL} / \mathrm{kg}$ delivered a $0.05 \mathrm{~mL} /$ $\mathrm{kg} / \mathrm{h}$ background infusion and $0.1 \mathrm{~mL} / \mathrm{kg}$ on-demand boluses with a 30-minute lockout time. Then, the PCA device bolus button was pushed once, and intravenous fentanyl $1.0 \mu \mathrm{g} /$ $\mathrm{kg}$ was injected. Immediately after PACU arrival, the bolus button was pushed once more. The PCA was accessible for 48 hours postoperatively.

\section{Postoperative management and patient assessments}

Postoperative pain was assessed using the revised Face, Legs, Activity, Cry, and Consolability (r-FLACC) pain scale. ${ }^{12}$ This scale consists of five subgroups (face, legs, activity, cry, and consolability) for which the child can be assigned 0,1 , or 2 points related to specific pain reactions, and an overall score of 7-10 indicates severe pain or discomfort. Preoperatively, the parents or primary caregivers completed a questionnaire regarding the individual pain behavior of the child related to each r-FLACC subgroup, and these answers were added. In the PACU, rescue analgesics (intravenous fentanyl $0.5 \mu \mathrm{g}$ / $\mathrm{kg}$ ) were administered when the r-FLACC score was $>4$. If severe nausea or vomiting occurred, the patient was treated with metoclopramide $0.2 \mathrm{mg} / \mathrm{kg}$. Epidural analgesia was considered successful if the r-FLACC score was $\leq 3$ when departing the PACU. In the ward, patients continued to receive the epidural PCA and as a supplement, all patients were prescribed ibuprofen 5-7 mg/kg per os three times per day to maintain an r-FLACC score $\leq 4$. However, if the r-FLACC score was persistently $>4$ or on parents' request, rescue intravenous tramadol $1 \mathrm{mg} / \mathrm{kg}$ was given. If severe nausea or vomiting occurred, we treated patients with $0.2 \mathrm{mg} / \mathrm{kg}$ metoclopramide. If dizziness, urinary retention, or intense motor blockade occurred, PCA was stopped temporarily. The PCA restarted only after these symptoms had been resolved. If the block was clinically inadequate or the epidural catheter was inadvertently dislodged, the patient was withdrawn from the study, and fentanyl-based intravenous PCA was prescribed.
Postoperative r-FLACC pain scores were our primary outcome. Scores were assessed 30 minutes after arrival at the PACU and 6, 12, 24, and 48 hours postoperatively. Secondary outcomes included analgesic consumption and adverse events. To assess epidural ropivacaine consumption, the amounts of epidural ropivacaine given as boluses and boluses plus background infusion, respectively, were recorded during 0-6, 6-12, 6-24, and 24-48 hours postoperatively. Cumulative doses of ropivacaine given as boluses and boluses plus background infusion, respectively, were also recorded at 6 , 12, 24, and 48 hours postoperatively. Finally, we recorded both the numbers of patients requiring additional rescue analgesics and the amounts of rescue analgesics used during $0-6,6-12,12-24$, and $24-48$ hours postoperatively. The consumption of fentanyl and tramadol was converted to intravenous morphine equivalents using the GlobalRPh morphine equivalence calculator assuming no cross-tolerance, accessed at http://globalrph.com/medcalcs/opioid-conversions-calcoriginal-single-agent. Adverse events such as postoperative nausea and vomiting, urinary retention, and motor blockade were recorded. The following perioperative data were also collected: MAP and HR (5 minutes after intubation [baseline], 5 minutes after skin incision, 1 hour after skin incision, immediately after skin closure, immediately after extubation, and 30 minutes after PACU admission), intraoperative blood loss, anesthesia time, and length of PACU stay. An investigator blinded to the group allocation collected all outcomes and perioperative data.

\section{Statistical analysis}

Distribution normality was assessed using KolmogorovSmirnov and Shapiro-Wilk tests. Continuous variables were expressed as mean \pm SD if data were normally distributed and median (interquartile range) if not. Categorical variables were expressed as absolute number (\%). Groups were compared on normally distributed data with an independent $t$-test, nonnormally distributed data with the Mann-Whiney $U$ test, and categorical variables with the chi-squared test or Fisher's exact test. The effects of preemptive epidural analgesia on both r-FLACC pain scores and the amount of rescue analgesics used were each assessed using a nonparametric mixed model provided by Brunner and Langer. ${ }^{13}$ Group differences in the amounts of epidural ropivacaine given as boluses and boluses plus background infusion, respectively, were assessed using a linear mixed model with patient indicator as a random effect and group, time, and group-by-time interaction as fixed effects. Group differences in cumulative doses of ropivacaine given as boluses and boluses plus background 
infusion, respectively, were similarly assessed using a linear mixed model. This method was also used to assess group differences in MAP and HR. These linear mixed model analyses used an unstructured covariance structure. The numbers of patients requiring additional rescue analgesics were compared between groups using a generalized estimation equation. $P$-values were corrected with Bonferroni methods for multiple comparisons.

Sample size was estimated based on previous data from our hospital, reporting a 1.7 SD for r-FLACC scores 12 hours postoperatively in this patient population. ${ }^{14}$ Assuming $\alpha=0.05$ and $\beta=0.15$ for a 1.5 -point difference in the r-FLACC scores between groups, the calculated sample size was 23 patients per group. To allow for a $10 \%$ dropout rate, the ideal sample size was 25 patients per group. All statistical analyses were performed using SPSS (version 20; IBM Corporation, Armonk, NY, USA) and SAS (version 9.2; SAS Institute Inc., Cary, NC, USA). A $P$-value $<0.05$ was considered statistically significant.

\section{Results}

Of the 61 patients assessed for eligibility, 11 were excluded due to age criteria $(n=5)$, current regular use of analgesics $(n=1)$, severe-profound cognitive impairment $(n=1)$, and refusal to participate $(n=4)$. Thus, 50 subjects were enrolled. Three were excluded from the analysis due to persistent motor and sensory block ( $n=1$, preemptive group), change of operation plan ( $\mathrm{n}=1$, preemptive group), and unexpected intensive care unit admission ( $n=1$, control group). Therefore, 47 patients were included in the final analysis (Figure 1). Patient characteristics and perioperative details were not significantly different between groups (Table 1).

Postoperative r-FLACC pain scores are demonstrated in Table 2. For r-FLACC pain scores, the group-by-time interaction was significant $(P=0.035)$. They were significantly lower in the preemptive group at 30 minutes after PACU arrival and 6 hours postoperatively (median difference $-1.0,95 \%$ $\mathrm{CI}-2.0$ to $-1.0, P=0.001$ and median difference $-2.0,95 \%$ $\mathrm{CI}-3.0$ to $-1.0, P=0.005$, respectively). However, they were not significantly different in both groups at 12,24 , and 48 hours postoperatively (median difference $0.0,95 \% \mathrm{CI}-1.0$ to $0.0, P>0.999$; median difference $-1.0,95 \% \mathrm{CI}-1.0$ to 0.0 , $P=0.692$; and median difference $-1.0,95 \% \mathrm{CI}-1.0$ to 0.0 , $P=0.351$, respectively).

Throughout 48 hours postoperatively, there were no group-by-time interactions for the amounts of epidural

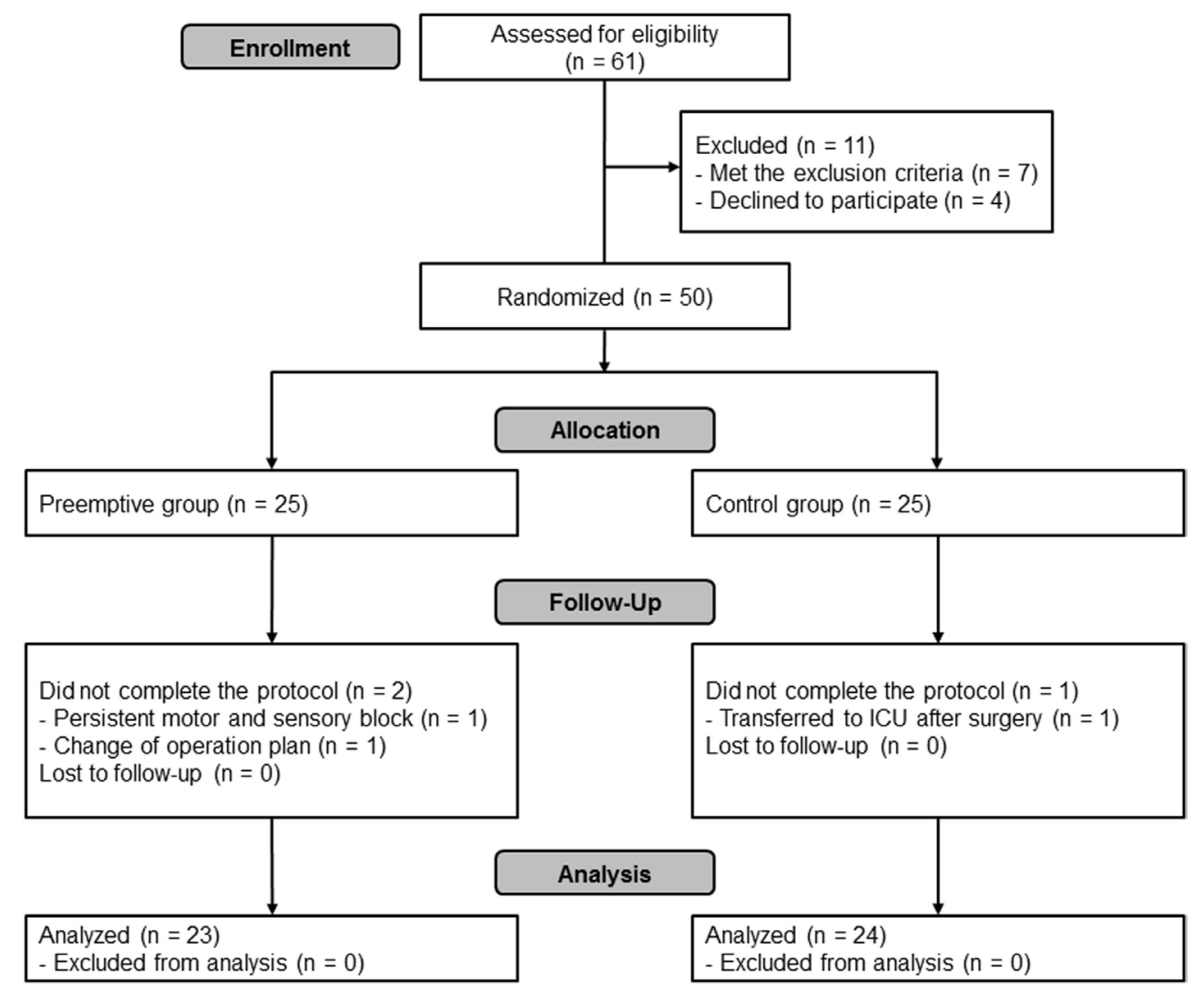

Figure I Flow diagram of patient selection.

Abbreviation: ICU, intensive care unit. 
Table I Patient characteristics and perioperative details

\begin{tabular}{|c|c|c|c|}
\hline & Preemptive $(n=23)$ & Control $(n=24)$ & $P$-value \\
\hline Female & $12(52.2)$ & $12(50.0)$ & 0.882 \\
\hline Age (years) & $10.0 \pm 1.7$ & $9.3 \pm 2.5$ & 0.300 \\
\hline Height (cm) & $\mid 33.4 \pm 14.8$ & $\mid 28.7 \pm 18.4$ & 0.370 \\
\hline Weight (kg) & $31.1 \pm 9.4$ & $29.5 \pm 11.1$ & 0.606 \\
\hline BMI $\left(\mathrm{kg} / \mathrm{m}^{2}\right)$ & $17.1 \pm 3.0$ & $17.3 \pm 3.9$ & 0.858 \\
\hline Previous surgery on the lower limbs & $9(39.1)$ & $8(33.3)$ & 0.679 \\
\hline Diagnosis & & & $>0.999$ \\
\hline Cerebral palsy & $21(91.3)$ & $21(87.5)$ & \\
\hline Others & $2(8.7)$ & $3(12.5)$ & \\
\hline Surgical procedure & $(n=46$ legs $)$ & $(n=48$ legs $)$ & 0.447 \\
\hline Pelvic osteotomy & II (24.I) & $15(31.3)$ & \\
\hline Femur osteotomy $y^{a}$ & $32(69.6)$ & $37(77.1)$ & \\
\hline Tibial osteotomy & $5(10.9)$ & $9(18.8)$ & \\
\hline Foot bony procedure & $10(21.7)$ & $6(12.5)$ & \\
\hline Capsulotomy of hip & I (2.2) & $I(2.1)$ & \\
\hline Open reduction of hip dislocation & $5(10.9)$ & $8(16.7)$ & \\
\hline Epiphysiodesis (femur or tibia tuberosity) & $9(19.6)$ & II (22.9) & \\
\hline Psoas lengthening & II (23.9) & $4(8.3)$ & \\
\hline Rectus femoris tenotomy & I (2.2) & $0(0.0)$ & \\
\hline Adductor tenotomy & $14(30.4)$ & $26(54.2)$ & \\
\hline Medial hamstring lengthening & $5(10.9)$ & $10(20.8)$ & \\
\hline Patella tendon advancement & $16(34.8)$ & $17(35.4)$ & \\
\hline Tibialis anterior transfer & $2(4.3)$ & I $(2 . I)$ & \\
\hline Tibialis posterior interventions & $3(6.5)$ & $I(2.1)$ & \\
\hline Calf muscle lengthening & $10(21.7)$ & $14(29.2)$ & \\
\hline Tendon achilles lengthening & $10(21.7)$ & $5(10.4)$ & \\
\hline Foot tendon interventions & $7(15.2)$ & $9(18.8)$ & \\
\hline Amount of intraoperative ropivacaine $(\mathrm{mg} / \mathrm{kg})$ & $1.01 \pm 0.3$ & - & - \\
\hline Intraoperative blood loss (mL) & $200.0(100.0-350.0)$ & $250.0(200.0-387.5)$ & 0.198 \\
\hline Total dose of ephedrine (mg) & $0.0(0.0-4.0)$ & $0.0(0.0-1.8)$ & 0.520 \\
\hline Total dose of phenylephrine $(\mu \mathrm{g})$ & $0.0(0.0-250.0)$ & $0.0(0.0-350.0)$ & 0.861 \\
\hline Operation time (minutes) & $269.3 \pm 92.6$ & $303.3 \pm 126.0$ & 0.299 \\
\hline Anesthesia time (minutes) & $334.8 \pm 102.6$ & $367.3 \pm 120.3$ & 0.325 \\
\hline Length of PACU stay (minutes) & $45.0(40.0-50.0)$ & $40.0(40.0-50.0)$ & 0.440 \\
\hline
\end{tabular}

Notes: Data are presented as mean \pm SD, median (interquartile range), or number of patients (\%). ${ }^{2}$ Femur osteotomy: femoral derotational osteotomy, femoral varizationderotational osteotomy, or distal femoral extension and shortening osteotomy.

Abbreviation: PACU, postanesthesia care unit.

Table 2 Postoperative pain scores determined using the r-FLACC pain scale

\begin{tabular}{|c|c|c|c|c|}
\hline & $\begin{array}{l}\text { Preemptive } \\
(n=23)\end{array}$ & $\begin{array}{l}\text { Control } \\
(n=24)\end{array}$ & $\begin{array}{l}\text { Median } \\
\text { difference } \\
(95 \% \mathrm{Cl})\end{array}$ & $P$-value \\
\hline$r-F L A C C$ & & & & $0.035^{a}$ \\
\hline PACU & $0.0(0.0-1.0)$ & $1.5(1.0-2.5)$ & $-1.0(-2.0$ to -1.0$)$ & 0.001 \\
\hline 6 hours & $1.0(0.0-2.0)$ & $3.0(2.0-4.0)$ & $-2.0(-3.0$ to -1.0$)$ & 0.005 \\
\hline 12 hours & $1.0(1.0-3.0)$ & $2.0(1.0-3.0)$ & $0.0(-1.0$ to 0.0$)$ & $>0.999$ \\
\hline 24 hours & $1.0(0.0-2.0)$ & $2.0(1.0-3.0)$ & $-1.0(-1.0$ to 0.0$)$ & 0.692 \\
\hline 48 hours & $0.0(0.0-1.0)$ & $1.0(0.5-2.0)$ & $-1.0(-1.0$ to 0.0$)$ & 0.351 \\
\hline
\end{tabular}

Notes: Data are presented as median (interquartile range). ${ }^{a p-v a l u e ~ o f ~ t h e ~ g r o u p-~}$ by-time interaction in the nonparametric mixed model.

Abbreviations: PACU, postanesthesia care unit; r-FLACC, revised Face, Legs, Activity, Cry, and Consolability.

ropivacaine given as boluses and boluses plus background infusion $(P=0.781$ and $P=0.892$, respectively; Table 3). Similarly, when all time points were combined, the cumulative doses of ropivacaine given as boluses and boluses plus background infusion did not significantly differ between groups ( $P=0.804$ and $P=0.816$, respectively). The numbers of patients requiring additional rescue analgesics and the amounts of rescue analgesics in terms of equianalgesic doses of morphine $(\mathrm{mg})$ were also not significantly different between groups over time ( $P=0.380$ and $P=0.261$, respectively).

The change of MAP and HR from baseline for both groups was consistent over time ( $P=0.144$ and $P=0.087$, respectively; Figure 2). However, comparing within groups at each time point revealed that HR was significantly lower in the preemptive group than in the control group throughout intraoperative epidural infusion. There were no significant differences in the incidence of adverse events between the two groups (Table 4). 
Table 3 Consumption of epidural ropivacaine by patient-controlled analgesia and additional analgesic requirements

\begin{tabular}{|c|c|c|c|c|}
\hline & Preemptive $(n=23)$ & Control $(n=24)$ & $\begin{array}{l}\text { Mean or median } \\
\text { difference }(95 \% \mathrm{Cl})\end{array}$ & P-value \\
\hline \multicolumn{4}{|c|}{ Amount of ropivacaine given as boluses $(\mathrm{mg} / \mathrm{kg} / \mathrm{h})$} & $0.78 I^{\mathrm{a}}$ \\
\hline $0-6$ hours & $0.07 \pm 0.03$ & $0.08 \pm 0.04$ & $-0.02(-0.04$ to 0.01$)$ & 0.512 \\
\hline $6-12$ hours & $0.05 \pm 0.05$ & $0.07 \pm 0.04$ & -0.01 ( $(-0.04$ to 0.01$)$ & $>0.999$ \\
\hline 12-24 hours & $0.07 \pm 0.04$ & $0.08 \pm 0.04$ & -0.01 ( -0.03 to 0.01$)$ & $>0.999$ \\
\hline $24-48$ hours & $0.05 \pm 0.04$ & $0.06 \pm 0.04$ & $-0.00(-0.03$ to 0.02$)$ & $>0.999$ \\
\hline \multicolumn{4}{|c|}{ Amount of ropivacaine given as boluses plus background infusion $(\mathrm{mg} / \mathrm{kg} / \mathrm{h})$} & $0.892^{\mathrm{a}}$ \\
\hline $0-6$ hours & $0.12 \pm 0.03$ & $0.13 \pm 0.04$ & $-0.02(-0.04$ to -0.01$)$ & 0.582 \\
\hline $6-12$ hours & $0.10 \pm 0.05$ & $0.12 \pm 0.04$ & $-0.01(-0.04$ to 0.01$)$ & $>0.999$ \\
\hline 12-24 hours & $0.12 \pm 0.04$ & $0.12 \pm 0.04$ & -0.01 ( $(-0.03$ to 0.01$)$ & $>0.999$ \\
\hline 24-48 hours & $0.10 \pm 0.04$ & $0.11 \pm 0.04$ & -0.01 ( -0.03 to 0.02$)$ & $>0.999$ \\
\hline \multicolumn{4}{|c|}{ Cumulative dose of ropivacaine given as boluses $(\mathrm{mg} / \mathrm{kg})$} & $0.804^{\mathrm{a}}$ \\
\hline$\sim 6$ hours & $0.48 \pm 0.23$ & $0.59 \pm 0.27$ & -0.11 ( $(-0.26$ to 0.04$)$ & 0.566 \\
\hline$\sim 12$ hours & $0.80 \pm 0.47$ & $0.98 \pm 0.45$ & $-0.18(-0.45$ to 0.09$)$ & 0.732 \\
\hline$\sim 24$ hours & $1.60 \pm 0.93$ & $1.90 \pm 0.78$ & $-0.30(-0.81$ to 0.20$)$ & 0.928 \\
\hline$\sim 48$ hours & $2.84 \pm 1.63$ & $3.24 \pm 1.37$ & $-0.40(-1.28$ to 0.49$)$ & $>0.999$ \\
\hline \multicolumn{4}{|c|}{ Cumulative dose of ropivacaine given as boluses plus background infusion $(\mathrm{mg} / \mathrm{kg})$} & $0.816^{\mathrm{a}}$ \\
\hline$\sim 6$ hours & $0.83 \pm 0.23$ & $0.93 \pm 0.27$ & $-0.10(-0.25$ to 0.05$)$ & 0.693 \\
\hline$\sim 12$ hours & $1.45 \pm 0.48$ & $1.63 \pm 0.45$ & $-0.18(-0.45$ to 0.10$)$ & 0.822 \\
\hline$\sim 24$ hours & $2.85 \pm 0.94$ & $3.14 \pm 0.78$ & $-0.29(-0.80$ to 0.22$)$ & $>0.999$ \\
\hline$\sim 48$ hours & $5.24 \pm 1.66$ & $5.66 \pm 1.37$ & $-0.42(-1.31$ to 0.47$)$ & $>0.999$ \\
\hline \multicolumn{4}{|c|}{ Patients receiving rescue analgesics } & $0.380^{\mathrm{b}}$ \\
\hline $0-6$ hours & $4(17.4)$ & $7(29.1)$ & - & $>0.999$ \\
\hline $6-12$ hours & $10(43.5)$ & $9(37.5)$ & - & $>0.999$ \\
\hline 12-24 hours & $12(52.2)$ & $10(41.7)$ & - & $>0.999$ \\
\hline $24-48$ hours & $10(43.5)$ & $12(50.0)$ & - & $>0.999$ \\
\hline \multicolumn{4}{|c|}{ Amount of rescue analgesics converted into equianalgesic doses of morphine $(\mathrm{mg})$} & $0.26 \mathrm{I}^{\mathrm{c}}$ \\
\hline $0-6$ hours & $0.00(0.00-0.00)$ & $0.00(0.00-0.03)$ & $0.00(0.00-0.00)$ & $>0.999$ \\
\hline $6-12$ hours & $0.00(0.00-0.03)$ & $0.00(0.00-0.03)$ & $0.00(0.00-0.00)$ & $>0.999$ \\
\hline $12-24$ hours & $0.03(0.00-0.03)$ & $0.00(0.00-0.03)$ & $0.00(0.00-0.03)$ & $>0.999$ \\
\hline 24-48 hours & $0.00(0.00-0.03)$ & $0.02(0.00-0.03)$ & $0.00(-0.03$ to 0.00$)$ & $>0.999$ \\
\hline
\end{tabular}

Notes: Data are presented as mean $\pm S D$, median (interquartile range), or number of patients (\%). a $P$-value of the group-by-time interaction in the linear mixed model. ${ }^{b} P$ value of the group-by-time interaction in the analysis using generalized estimation equation. ${ }^{c} P$-value of the group-by-time interaction in the nonparametric mixed model.

\section{Discussion}

This study demonstrated that in this patient population, intraoperative epidural ropivacaine infusion significantly lowered pain scores relative to saline treatment only up to 6 hours postoperatively, but not at 12, 24, and 48 hours postoperatively. Additionally, epidural ropivacaine consumption and additional analgesic requirements during postoperative 48 hours were not significantly different between the groups.

Pediatric patients undergoing extensive lower limb orthopedic surgery experience frequent and severe pain postoperatively. ${ }^{1}$ Although animal studies on preemptive analgesic efficacy yielded promising results, ${ }^{4}$ clinical research on this subject in pediatric patients is limited. In a recent study, Song et al reported that for pediatric patients undergoing corrective osteotomy, preemptive analgesia using intravenous PCA with fentanyl did not significantly affect postoperative pain. ${ }^{15}$ However, this study had two key limitations that may explain these negative results. First, they compared the effects of PCA when initiated either just before or after skin incision, conforming to the classic view of preemptive analgesia. Second, they used a systemic opioid, which may not sufficiently block nociceptive input to prevent central sensitization. ${ }^{16}$

Preventive analgesia, a broader approach than the restrictive definition of preemptive analgesia, minimizes sensitization induced by noxious perioperative stimuli, including those arising preoperatively, intraoperatively, and postoperatively. ${ }^{8,17}$ Considering that multiple skin incisions and/or osteotomy during single-event multilevel surgery could produce strong and continuous nociceptive input, this broader approach, especially focused on the intraoperative period, could be more effective than antinociceptive procedures implemented only preoperatively..$^{18}$ Additionally, in a previous study comparing the effects of epidural anesthesia alone, general anesthesia, or combined epidural and general 

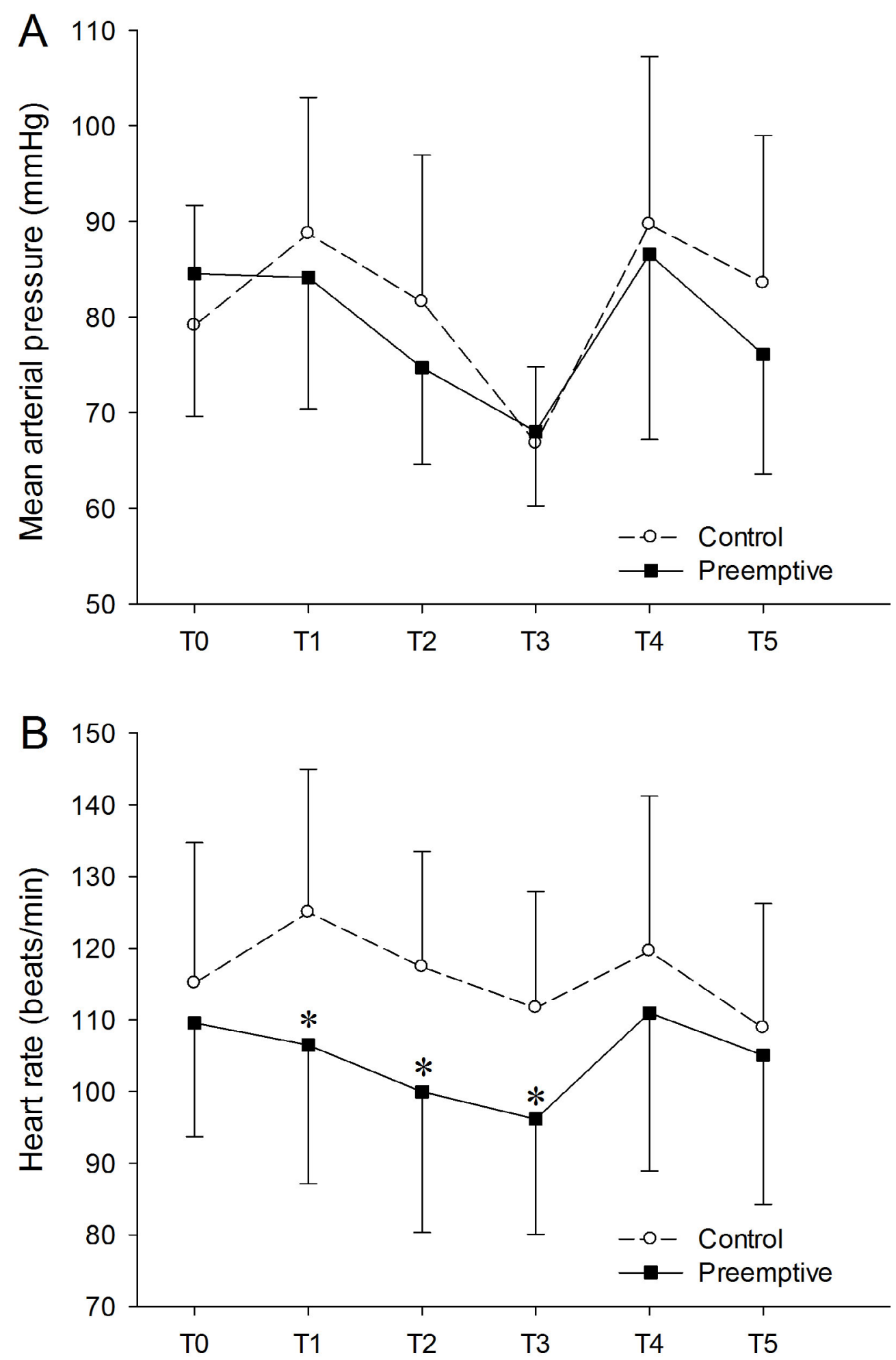

Figure 2 Perioperative hemodynamic variables.

Notes: (A) Mean arterial pressure did not significantly differ between treatment groups at any time point. (B) Heart rate was significantly lower for the preemptive treatment group relative to control group between $\mathrm{TI}$ and T3. All values are mean \pm SD. $* P<0.05$ vs control group. T0, 5 minutes after intubation (baseline); TI, 5 minutes after skin incision; T2, I hour after skin incision; T3, immediately after skin closure; $\mathrm{T} 4$, immediately after extubation; $\mathrm{T} 5,30$ minutes after postanesthesia care unit admission.

anesthesia on postoperative pain and analgesia in patients undergoing radical prostatectomy, improved postoperative analgesia was observed in patients with epidural anesthesia alone. ${ }^{19}$ This suggests that epidural intraoperative analgesics can sufficiently block nociceptive inputs. However, in previous reports on the efficacy of epidural analgesia for postoperative pain control in children undergoing major orthopedic surgery of the lower extremities, local anesthetic 
Table 4 Frequency of adverse events

\begin{tabular}{|l|l|l|l|}
\hline & $\begin{array}{l}\text { Preemptive } \\
(\mathbf{n = 2 3 )}\end{array}$ & $\begin{array}{l}\text { Control } \\
(\mathbf{n = 2 4 )}\end{array}$ & P-value \\
\hline PONV & $\mathrm{II}(47.8)$ & $9(37.5)$ & 0.474 \\
$\begin{array}{l}\text { Temporary } \\
\text { discontinuation of PCA }\end{array}$ & $3(13.0)$ & $\mathrm{I}(4.2)$ & 0.348 \\
$\begin{array}{l}\text { Urinary retention } \\
\begin{array}{l}\text { Transient motor } \\
\text { blockade }\end{array}\end{array}$ & $4(17.4)$ & $\mathrm{I}(4.2)$ & 0.188 \\
Headache & $\mathrm{I}(4.3)$ & $3(12.5)$ & 0.609 \\
\hline
\end{tabular}

Note: Data are presented as number of patients (\%).

Abbreviations: PCA, patient-controlled analgesia; PONV, postoperative nausea and vomiting.

infusion was started 1 hour before the end of the anesthesia or postoperative period. ${ }^{1,10,20}$

Thus, the authors tested whether intraoperative epidural infusion of ropivacaine could alleviate acute postoperative pain in pediatric patients undergoing extensive lower limb orthopedic surgery. This treatment only significantly lowered pain scores within the first 6 hours postoperatively, and otherwise did not significantly affect analgesic outcomes. According to Katz et al, preventive analgesia occurs when postoperative pain is still reduced after the duration of action of the target drug, or after 5.5 half-lives of the target drug. ${ }^{8}$ Based on previous reports, we estimate that at the concentration and volume administered in this study, the half-life of ropivacaine is $\sim 3$ hours. ${ }^{21-24}$ Therefore, our study outcomes may result from the direct analgesic effect of intraoperative epidural infusion and not preemptive analgesia.

There are many possible explanations for the lack of preemptive analgesic efficacy in this study. First, intraoperative epidural infusion of ropivacaine may not sufficiently block the overwhelming nociceptive input. Importantly, favorable effects of preemptive analgesia have been reported in adult patients undergoing radical prostatectomy when there was sufficient epidural anesthesia, which was verified by measuring the sensory level before general anesthesia induction and in the PACU. ${ }^{25}$ In that study, patients who did not have a T4 sensory level were excluded. In the present study, the epidural catheter was placed following anesthetic induction, and the cast was applied after surgery (eg, spica cast). Thus, it was not possible to precisely assess the sensory blockade of intraoperative epidural infusion in the PACU. However, the epidural infusion regimen in this study was modified according to the epidural analgesia protocol for children in our hospital. The concentration and volume of epidural ropivacaine for children were based on several references., ${ }^{3,14,22}$ In addition, as HR was significantly higher in the control group than in the preemptive group throughout intraopera- tive epidural infusion, there was enough blockade to prevent pain-induced increases in sympathetic tone. Importantly, excessive blockade of spinal nociception could impede the detection of critical side effects of extensive lower limb orthopedic surgery, such as nerve injury or compartment syndrome. Thus, we could not intraoperatively infuse high doses of ropivacaine while expecting only preemptive analgesia. Therefore, our results do not disapprove preemptive analgesia itself, but signify that intraoperative epidural infusion of local anesthetic alone does not achieve effective preemptive analgesia in this patient population. Second, the combination of surgical procedures that the patients underwent varied greatly. Despite this inter-individual variability, surgical procedure details were not statistically different between groups (Table 1). Additionally, we assumed that the regimen of intraoperative epidural infusion produced sensory blockade up to the T12 level, which covers most of the surgical site. Third, our study did not consider the extent to which postoperative peripheral nociceptive inputs contribute to central sensitization and postoperative pain. Gordon et al demonstrated that in patients undergoing third molar extraction surgery, the peripheral nociceptive barrage occurring after the surgery contributes to central sensitization more extensively than the nociceptive barrage occurring intraoperatively. ${ }^{26}$ In Kjeldgaard Pedersen et al's study, which tested the efficacy of epidural analgesia and local infiltration analgesia for early postoperative pain control in children undergoing unilateral hip reconstruction, the patients of the epidural group received continuous epidural infusion of local anesthetic during the anesthesia, which was maintained for the first 2-3 days postoperatively. ${ }^{27}$ Although direct comparison is not possible, the postoperative r-FLACC pain scores in this study were rather slightly lower than in Kjeldgaard Pedersen et al's study. Further controlled studies will be needed focusing on this issue.

\section{Limitations}

Our study had a few limitations. First, we used epidural PCA with a background infusion, which might affect postoperative outcomes. However, the background infusion rate of $0.05 \mathrm{mg} / \mathrm{kg} / \mathrm{h}$ was considerably lower than the postoperative epidural ropivacaine infusion rate recommended to children over 6 months. ${ }^{24,28}$ Second, intravenous fentanyl was administered during the intraoperative period. However, the same dose of fentanyl was used in both groups, and the likelihood of opioid-induced hyperalgesia from $2.0 \mu \mathrm{g} / \mathrm{kg}$ fentanyl is slight. ${ }^{29}$

Overall, our results reveal that preemptive analgesia should focus on the prevention of central sensitization 
along with the blockade of afferent nociceptive inputs. In this context, it would be interesting to study the efficacy of preemptive analgesia for this patient population using an $N$-methyl-D-aspartate receptor antagonist, which could attenuate central sensitization as a supplement to intraoperative epidural infusion of local anesthetic. ${ }^{30}$ In addition, multimodal analgesia is regarded as the most successful and powerful method to improve outcomes after surgery. ${ }^{31}$ In a recent animal study about multimodal preemptive analgesic strategy, the addition of preemptive ketamine-lidocaine infusion to single intravenous dose of tramadol enhanced the attenuation of central sensitization and improved intraoperative and postoperative analgesia in dogs undergoing ovariohysterectomy. ${ }^{32}$ Therefore, our results suggest that preemptive analgesia should be included in multimodal analgesia rather than as a single method.

\section{Conclusion}

Intraoperative epidural infusion of ropivacaine in pediatric patients undergoing major orthopedic surgery of the lower extremities reduced pain scores only up to 6 hours postoperatively and not thereafter. Additionally, postoperative analgesic consumption during 48 hours postoperatively was not significantly different between groups. This lack of preemptive analgesic efficacy emphasizes the importance of multimodal analgesia for this patient population.

\section{Disclosure}

The authors report no conflicts of interest in this work.

\section{References}

1. Løvstad RZ, Støen R. Postoperative epidural analgesia in children after major orthopaedic surgery. A randomised study of the effect on PONV of two anaesthetic techniques: low and high dose i.v. fentanyl and epidural infusions with and without fentanyl. Acta Anaesthesiol Scand. 2001;45(4):482-488.

2. Nowicki PD, Vanderhave KL, Gibbons K, et al. Perioperative pain control in pediatric patients undergoing orthopaedic surgery. $\mathrm{J} \mathrm{Am} \mathrm{Acad}$ Orthop Surg. 2012;20(12):755-765.

3. Lönnqvist PA, Morton NS. Postoperative analgesia in infants and children. Br J Anaesth. 2005;95(1):59-68.

4. Kissin I. Preemptive analgesia. Why its effect is not always obvious. Anesthesiology. 1996;84(5):1015-1019.

5. Møiniche S, Kehlet H, Dahl JB. A qualitative and quantitative systematic review of preemptive analgesia for postoperative pain relief: the role of timing of analgesia. Anesthesiology. 2002;96(3):725-741.

6. Obata H, Saito S, Fujita N, Fuse Y, Ishizaki K, Goto F. Epidural block with mepivacaine before surgery reduces long-term post-thoracotomy pain. Can J Anaesth. 1999;46(12):1127-1132.

7. Flisberg P, Törnebrandt K, Walther B, Lundberg J. A comparison of the effects on postoperative pain relief of epidural analgesia started before or after surgery. Eur J Anaesthesiol. 2000;17(10):627-633.

8. Katz J, Clarke H, Seltzer Z. Review article: preventive analgesia: quo vadimus? Anesth Analg. 2011;113(5):1242-1253.

9. Kissin I. Preemptive analgesia. Anesthesiology. 2000;93(4):1138-1143.
10. Nolan J, Chalkiadis GA, Low J, Olesch CA, Brown TC. Anaesthesia and pain management in cerebral palsy. Anaesthesia. 2000;55(1):32-41.

11. Ong CK, Lirk P, Seymour RA, Jenkins BJ. The efficacy of preemptive analgesia for acute postoperative pain management: a meta-analysis. Anesth Analg. 2005;100(3):757-773.

12. Malviya S, Voepel-Lewis T, Burke C, Merkel S, Tait AR. The revised FLACC observational pain tool: improved reliability and validity for pain assessment in children with cognitive impairment. Paediatr Anaesth. 2006;16(3):258-265.

13. Brunner E, Langer F. Nonparametric analysis of ordered categorical data in designs with longitudinal observations and small sample sizes. Biom J. 2000;42(6):663-675.

14. Park SJ, Shin S, Kim SH, et al. Comparison of dexmedetomidine and fentanyl as an adjuvant to ropivacaine for postoperative epidural analgesia in pediatric orthopedic surgery. Yonsei Med J. 2017;58(3):650-657.

15. Song IK, Park YH, Lee JH, Kim JT, Choi IH, Kim HS. Randomized controlled trial on preemptive analgesia for acute postoperative pain management in children. Paediatr Anaesth. 2016;26(4):438-443.

16. Katz J, Clairoux M, Redahan C, et al. High dose alfentanil pre-empts pain after abdominal hysterectomy. Pain. 1996;68(1):109-118.

17. Kissin I. Preemptive analgesia: terminology and clinical relevance. Anesth Analg. 1994;79(4):809-810.

18. Gordon SM, Dionne RA, Brahim J, Jabir F, Dubner R. Blockade of peripheral neuronal barrage reduces postoperative pain. Pain. 1997;70(2-3):209-215.

19. Shir Y, Raja SN, Frank SM. The effect of epidural versus general anesthesia on postoperative pain and analgesic requirements in patients undergoing radical prostatectomy. Anesthesiology. 1994;80(1):49-56.

20. Brenn BR, Brislin RP, Rose JB. Epidural analgesia in children with cerebral palsy. Can J Anaesth. 1998;45(12):1156-1161.

21. Ivani G, Lampugnani E, de Negri P, Lonnqvist PA, Broadman L. Ropivacaine vs bupivacaine in major surgery in infants. Can JAnaesth. 1999;46(5 Pt 1):467-469.

22. Hansen TG, Ilett KF, Lim SI, Reid C, Hackett LP, Bergesio R. Pharmacokinetics and clinical efficacy of long-term epidural ropivacaine infusion in children. Br J Anaesth. 2000;85(3):347-353.

23. Mcclellan KJ, Faulds D. Ropivacaine: an update of its use in regional anaesthesia. Drugs. 2000;60(5):1065-1093.

24. Simpson D, Curran MP, Oldfield V, Keating GM. Ropivacaine: a review of its use in regional anaesthesia and acute pain management. Drugs. 2005;65(18):2675-2717.

25. Gottschalk A, Smith DS, Jobes DR, et al. Preemptive epidural analgesia and recovery from radical prostatectomy: a randomized controlled trial. JAMA. 1998;279(14):1076-1082.

26. Gordon SM, Brahim JS, Dubner R, Mccullagh LM, Sang C, Dionne RA. Attenuation of pain in a randomized trial by suppression of peripheral nociceptive activity in the immediate postoperative period. Anesth Analg. 2002;95(5):1351-1357.

27. Kjeldgaard Pedersen L, Nikolajsen L, Rahbek O, Uldall Duch B, MøllerMadsen B. Epidural analgesia is superior to local infiltration analgesia in children with cerebral palsy undergoing unilateral hip reconstruction. Acta Orthop. 2016;87(2):176-182.

28. Brasher C, Gafsous B, Dugue S, et al. Postoperative pain management in children and infants: an update. Pediatr Drugs. 2014;16(2):129-140.

29. Mauermann E, Filitz J, Dolder P, Rentsch KM, Bandschapp O, Ruppen W. Does fentanyl lead to opioid-induced hyperalgesia in healthy volunteers?: A double-blind, randomized, crossover trial. Anesthesiology. 2016;124(2):453-463.

30. Mccartney CJ, Sinha A, Katz J. A qualitative systematic review of the role of $\mathrm{N}$-methyl-D-aspartate receptor antagonists in preventive analgesia. Anesth Analg. 2004;98(5):1385-1400.

31. Russell P, von Ungern-Sternberg BS, Schug SA. Perioperative analgesia in pediatric surgery. Curr Opin Anaesthesiol. 2013;26(4):420-427.

32. Kaka U, Rahman NA, Abubakar AA, et al. Pre-emptive multimodal analgesia with tramadol and ketamine-lidocaine infusion for suppression of central sensitization in a dog model of ovariohysterectomy. J Pain Res. 2018;11:743-752. 
The Journal of Pain Research is an international, peer reviewed, open access, online journal that welcomes laboratory and clinical findings in the fields of pain research and the prevention and management of pain. Original research, reviews, symposium reports, hypothesis formation and commentaries are all considered for publication.
The manuscript management system is completely online and includes a very quick and fair peer-review system, which is all easy to use. Visit http://www.dovepress.com/testimonials.php to read real quotes from published authors. 